7-19-2021

\title{
Does Exercise Prevent Gestational Diabetes Mellitus in Pregnant Women? A Clin-IQ
}

Olivia Lust

Tana Chongsuwat

Elizabeth Lanham

Ann F. Chou

Elizabeth Wickersham

Follow this and additional works at: https://aah.org/jpcrr

Part of the Endocrinology, Diabetes, and Metabolism Commons, Family Medicine Commons, Female Urogenital Diseases and Pregnancy Complications Commons, Health and Medical Administration Commons, and the Obstetrics and Gynecology Commons

\section{Recommended Citation}

Lust O, Chongsuwat T, Lanham E, Chou AF, Wickersham E. Does exercise prevent gestational diabetes mellitus in pregnant women? A Clin-IQ. J Patient Cent Res Rev. 2021;8:281-5. doi: 10.17294/

2330-0698.1811

Published quarterly by Midwest-based health system Advocate Aurora Health and indexed in PubMed Central, the Journal of Patient-Centered Research and Reviews (JPCRR) is an open access, peer-reviewed medical journal focused on disseminating scholarly works devoted to improving patient-centered care practices, health outcomes, and the patient experience. 


\title{
Does Exercise Prevent Gestational Diabetes Mellitus in Pregnant Women? A Clin-IQ
}

\author{
Olivia Lust, DO, Tana Chongsuwat, MD, Elizabeth Lanham, BS, Ann F. Chou, PhD, MPH, MA, \\ Elizabeth Wickersham, MD, MS \\ Department of Family and Preventive Medicine, University of Oklahoma College of Medicine, University of Oklahoma \\ Health Sciences Center, Oklahoma City, OK
}

\begin{abstract}
Excessive weight gain during pregnancy has been on the rise globally, leading to increased prevalence of gestational diabetes mellitus (GDM). A diagnosis of GDM often leads to pregnancy and infantrelated complications. Regular exercise may have the potential to prevent GDM. However, evidence surrounding the utility of exercise during pregnancy as an effective risk reduction intervention has been mixed. This clinical inquiry examined the role of regular exercise during pregnancy in preventing GDM in both obese and normal-weight women and analyzed specific aspects of exercise that make it an effective preventive measure. The review of evidence included 3 meta-analyses, 3 systematic reviews, and 1 umbrella review. Findings identified several components of an exercise program that can reduce GDM risk. Specifically, an exercise intervention of 40- to 60-minute sessions 3 times per week beginning as early as possible during pregnancy and continuing with good adherence over the course of pregnancy yielded clinically significant results. Adhering to a similar exercise routine before pregnancy also was shown to be protective against GDM for all women, but especially so for women who are overweight or obese. (J Patient Cent Res Rev. 2021;8:281-285.)
\end{abstract}

Keywords pregnancy; gestational diabetes; exercise; prevention; maternal health

\section{Clinical Question}

In pregnant women, does exercise as an intervention prevent gestational diabetes mellitus (GDM) compared to no intervention?

\section{Brief Answer}

Yes. Exercise can help reduce risk of GDM and should be recommended to women of child-bearing age. However, its success is dependent on several factors such as early initiation of intervention, correct intensity and frequency of exercise, and management of gestational weight gain. Recommendations include moderately intense physical activity 40-60 minutes per day at least 3 times per week before, during, and after pregnancy.

Level of Evidence for the Answer: B.

Date Answer Was Determined: September 21, 2020.

Corresponding author: Elizabeth Wickersham, MD, MS, Family and Preventive Medicine, University of Oklahoma Health Sciences Center, 900 NE 10th St., Oklahoma City, OK 73104 (elizabeth-wickersham@ouhsc.edu)
Literature Search

Databases: Ovid MEDLINE, PubMed, Cochrane Library.

Date of Search: July 20, 2020.

Exclusion Criteria: Non-English language, articles published before 2014, diagnosis of diabetes prior to pregnancy, article titles already included in meta-analyses or systematic reviews that were analyzed for this clinical inquiry (Clin-IQ).

Inclusion Criteria: Systematic reviews, meta-analyses, randomized controlled trials, cohort studies, and consensus guidelines published in the English language within the date range of 2014-2020.

Search Terms: Exercise, gestational diabetes, pregnancy.

\section{Summary of Issues}

In the United States $15 \%-20 \%$ of pregnancies are complicated by GDM, while other parts of the world have a prevalence up to $30 \% .{ }^{1}$ GDM is characterized by glucose intolerance occurring after 20 weeks gestational age in pregnancy. A diagnosis of GDM increases the risk of adverse birth outcomes for both mother and infant, such as preeclampsia, higher likelihood for caesarean delivery, infant macrosomia, neonatal hypoglycemia, and neonatal intensive care unit admission. Moreover, women diagnosed with GDM have up to a $70 \%$ chance 
of developing type 2 diabetes postpregnancy, while epigenetic changes to the affected infant may increase risk of development of type 2 diabetes, cardiovascular complications (eg, hypertension), and obesity later in life. ${ }^{2}$

Given these complications, much research has been conducted to identify interventions to reduce the risk of developing GDM and outline prevention recommendations for pregnant women. Known risk factors for GDM include advanced maternal age, history of GDM or fetal macrosomia in a prior pregnancy, and family history of diabetes. In particular, a significant factor contributing to this rise in GDM prevalence may be the concurrent rise in obesity, as obesity exaggerates insulin resistance in normal pregnancy caused by placental hormones. ${ }^{3}$ Excess adipose tissue also increases release of adipokines, chemokines, and cytokines, which in turn increase the risk of GDM. ${ }^{3}$

Exercise in individuals with diabetes has long been prescribed to help disease management by increasing insulin sensitivity and improving glycemic control. Exercise promotes an increase in glucose uptake by skeletal muscle through translocation of the insulinsensitive GLUT4 glucose transporter to the cell surface, thus lowering glucose levels and increasing insulin sensitivity. ${ }^{3,4}$ Based on this body of evidence, many medical professional groups recommend exercise during pregnancy to reduce the risk of developing GDM. For instance, the American College of Obstetricians and Gynecologists currently recommends aerobic and strength training exercises for women who have uncomplicated pregnancies, specifically, 30-60 minutes of moderately intense exercise 3-4 times per week throughout the pregnancy. ${ }^{5}$ However, few women achieve these exercise goals during pregnancy, with only $23 \%-29 \%$ of pregnant women in the United States meeting the minimum exercise guidelines. ${ }^{6}$

Despite the extensive research in this area, the exact type of exercise and specifics to determine the best exercise program remain unclear. This Clin-IQ article aims to review the relevant literature to determine an answer characterizing the relationship between exercise during pregnancy and prevention of GDM.

\section{Summary of the Evidence}

The literature search yielded 11 articles evaluating the effectiveness of exercise in reducing risk of GDM. In addition to 1 Cochrane review, 3 meta-analyses, 4 systematic reviews, 2 combinations of systematic review and meta-analysis, and 1 umbrella review were analyzed (Figure 1). While all 11 articles focused on exercise as an intervention for decreasing incidence of GDM, the intensity, duration, type of intervention, initiation of intervention, and participant population varied across studies. ${ }^{1,3,4,6-13}$

The Cochrane review analyzed data on GDM from 11 past Cochrane works to assess effects of different interventions - ranging from diet, exercise, or both to dietary supplements to pharmaceuticals - during pregnancy. Evidence on exercise alone (from 3 trials comprising 826 women) was deemed low quality and, compared to standard antenatal care, had an unclear effect on the risk of GDM (relative risk [RR]: 1.10, 95\% CI: $0.66-1.84){ }^{7}$ Of the other 10 review articles analyzed, all demonstrated clear benefits of exercise in reducing GDM risk, regardless of the timing of the initiation of exercise, intensity, and frequency.

In their 2018 meta-analysis, Mijatovic-Vukas and colleagues studied the impact of exercise on the risk of GDM and found that exercise was overall protective against GDM in 13 of 17 studies. ${ }^{1}$ Engaging in exercise during early pregnancy resulted in $21 \%$ reduced odds of developing GDM. The lowest risk of developing GDM was found in participants who engaged in exercise both before and during pregnancy (RR: $0.31,95 \% \mathrm{CI}$ : 0.12-0.79). This meta-analysis also evaluated how patient-reported Leisure Time Physical Activity (LTPA) correlates to GDM diagnosis - LTPA refers to all behavior connected with physical activity in which people engage as part of daily life - and found that 90 minutes per week in LTPA before pregnancy was associated with $46 \%$ reduced odds of GDM. ${ }^{1}$

Similar results describing benefits of exercise during pregnancy were reported in an umbrella review by DiPietro et al, ${ }^{6} 2$ other meta-analyses, ${ }^{8,9}$ and a combined systematic review and meta-analysis by Benette et al. ${ }^{10}$ In 2019, DiPietro and colleagues found that 8 of the 13 meta-analyses reviewed cited statistically significant reductions in risk of GDM, with a median RR of $0.73 .{ }^{6}$ The greatest overall reduced risk was found in women performing moderately intense exercise both before and during pregnancy, with a 59\% GDM risk reduction (RR: 0.41, 95\% CI: $0.23-0.73){ }^{6}$

Also published in 2019, a meta-analysis by Guo et al included 47 randomized controlled trials studying effects of diet, exercise, or both on GDM prevention. ${ }^{8}$ The assessed interventions began between 7 and 20 weeks gestational age and continued through delivery. The results showed that an intervention consisting of moderate-intensity exercise for 50-60 minutes twice a week resulted in a risk reduction of approximately $24 \%$ (RR: $0.70,95 \%$ CI: 0.59-0.84). Diet had a synergistic 


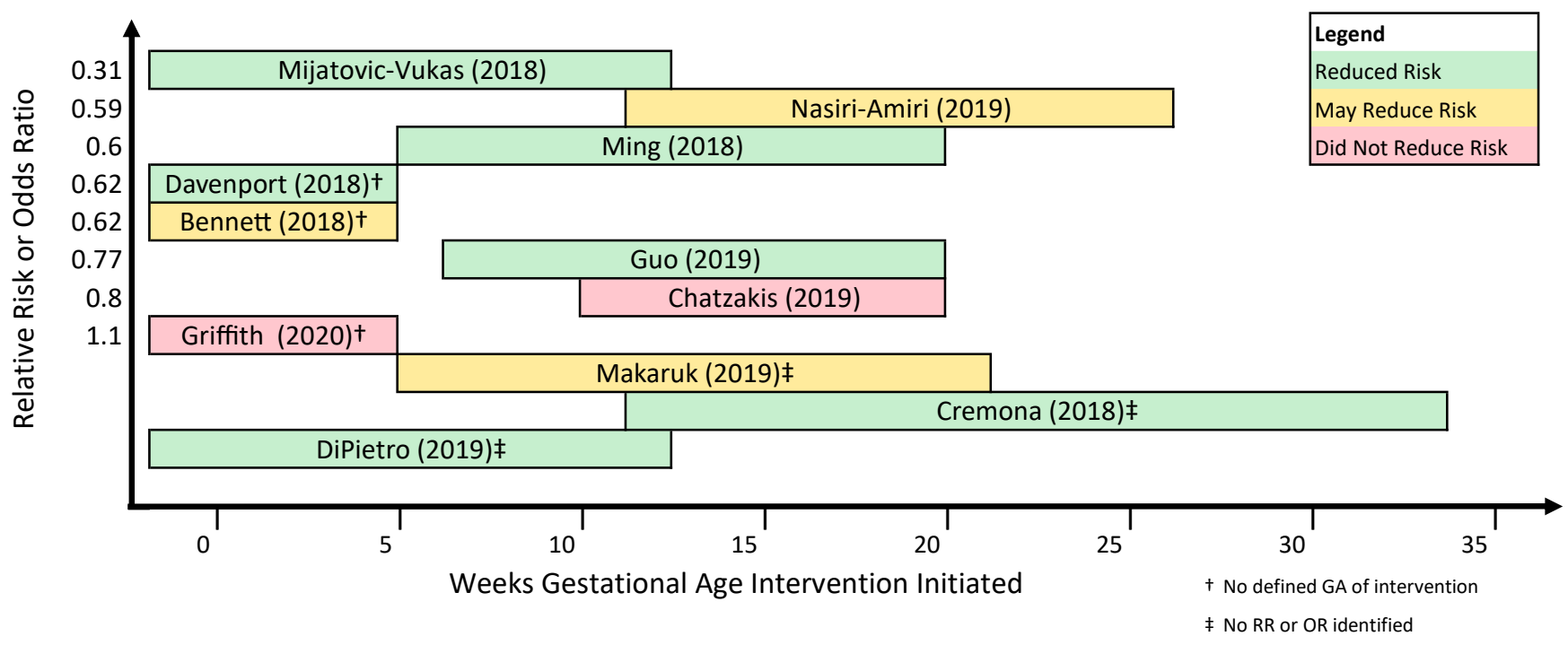

Figure 1. Summary of reviewed articles.

effect when combined with exercise to further improve risk reduction (RR: 0.77, 95\% CI: 0.69-0.87). Increasing that regimen to 3 times per week led to a $35 \%$ reduction in GDM risk. ${ }^{8}$ Similar to the conclusions reported by Mijatovic-Vukas et al's meta-analysis, ${ }^{1}$ Guo and colleagues found that the earlier an exercise intervention begins, the larger the reduction in GDM risk. ${ }^{8}$

A 2018 meta-anaylsis by Ming et al investigated the effect of exercise during pregnancy on the occurrence of GDM specifically in normal-weight women (prepregnancy body mass index between 18.5 and $\left.24.9 \mathrm{~kg} / \mathrm{m}^{2}\right) .{ }^{9}$ The 8 studies that met the inclusion criteria included 2981 pregnant women. The primary outcome studied was occurrence of GDM; secondary outcomes examined gestational weight gain, gestational age at birth, birth weight, and the odds of cesarean section. Occurrence of GDM was shown to decrease with planned, structured, repetitive movement during pregnancy (RR: $0.58,95 \%$ CI: 0.37-0.90). ${ }^{9}$ The findings demonstrated a protective effect of exercise against GDM risk and decreased gestational weight gain in normal-weight participants. Most importantly, this study showed that participating in exercise during pregnancy is beneficial for all women, not just those at high risk. ${ }^{9}$ These results are further supported by Bennett and colleagues' systematic review and meta-analysis of randomized controlled trials examining the effects of physical activity and lifestyle interventions implemented as early as 9 weeks (mean of 25 weeks) in gestational age, which found that physical activity alone reduced risk of GDM by $38 \%$ (RR: 0.62 , 95\% CI: 0.50-0.78; $\mathrm{P}<0.001) .{ }^{10}$
Two articles further examined aspects of exercise during pregnancy in an attempt to specify components of exercise that affected GDM risk. ${ }^{4,11}$ Findings from these studies were mixed. Cremona and colleagues conducted a systematic review to assess possible differential effects across exercise modalities on glycemic control during pregnancy. ${ }^{4}$ The review included 12 studies with 2 using resistance training, 8 using aerobic training, and 2 that combined both modalities. Total duration ranged from 5 to 24 weeks, with 6 starting at the time of GDM diagnosis (24-34 weeks gestational age), 1 starting in the first trimester, and the rest starting at 12-23 weeks gestational age. Results of the review found that exercise reduced GDM incidence in those at risk and reduced intervention requirements for those who had a diagnosis of GDM. ${ }^{4}$ The authors explained that both exercise modalities may have led to increased glycemic control by triggering metabolic pathways that lower blood glucose and improve insulin sensitivity. Aerobic exercise may be more effective at increasing glucose uptake into muscle and reducing fat mass, while resistance exercises help to increase lean muscle mass and increase basal metabolic rate. Both types of exercise did lead to lower incidence of GDM and better glycemic control for patients already diagnosed; however, the differences between them were difficult to determine due to the variance in study designs and reporting. ${ }^{4}$

A 2018 systematic review by Davenport et al included 106 studies $(\mathrm{N}=273,182$ subjects $)$ to estimate the effects of exercise on the odds of developing GDM. ${ }^{11}$ Frequency, duration, and types of exercise varied across 
studies, but in contrast to Cremona et al, a reduction of GDM was observed only in exercise-alone interventions ( $\mathrm{n}=6934$; odds ratio: $0.62,95 \%$ CI: $0.52-0.75$ ). Overall, 600 metabolic equivalent (MET) minutes per week of moderate-intensity exercise (eg, brisk walk, water aerobics, stationary cycling, resistance training) was found to be protective for pregnant women, with a $25 \%$ reduction in the odds of developing GDM. Benefits would be attained when exercise was performed for at least 25 minutes per session at a frequency of 2.1 days per week. ${ }^{11}$

Three studies specifically examined the effect of exercise on GDM risk among overweight and obese women during pregnancy. ${ }^{3,12,13}$ A 2019 network metaanalysis by Chatzakis and colleagues on the efficiency of multiple interventions in preventing GDM among obese/overweight pregnant women included randomized controlled trials that had both an intervention and placebo group and whose participants had a body mass index of $>25 \mathrm{~kg} / \mathrm{m}^{2} .{ }^{3}$ Of the 23 studies included ( $\mathrm{N}=4327$ patients), 13 used an exercise intervention, which varied by study in type of exercise, initiation of intervention, duration of program, and length per session. Exercise as an intervention was determined to be not superior to placebo/ no intervention, yet exercise was successful in reducing gestational weight gain. While it is counterintuitive that reducing gestational weight gain did not result in reduced GDM risk, as weight gain is a known risk factor for GDM, the authors concluded that the best timeline for prevention of GDM in overweight/obese women through exercise may actually occur preconception. ${ }^{3}$ According to one study cited, overweight women who reduced their preconception body mass index by $10 \%$ had a $25 \%$ risk reduction in developing GDM. ${ }^{14}$ It was proposed that by entering pregnancy in an already insulin-resistant state, overweight and obese women may have less protective benefits of exercise in GDM prevention as compared to normal-weight women.

In a 2019 systematic review and meta-analysis by NasiriAmiri and colleagues, the 8 studies included investigated the intervention of exercise versus a control group (no exercise) in obese and overweight pregnant women on the incidence of GDM. ${ }^{12}$ Exercise programs ranged in type, intensity, duration, and frequency as well as variability in time intervention started in pregnancy. Interestingly, interventions of 3 exercise periods per week or less were found to significantly reduce the incidence of GDM (RR: 0.59, 95\% CI: 0.46-0.76; $\mathrm{P}=0.47$ ); however, no difference was found between control groups and interventions that consisted of more than 3 exercise periods per week (RR: 1.03, 95\% CI: $0.78-1.35 ; \mathrm{P}=0.46$ ). Overall, there was no statistical significance of exercise on the incidence of GDM in the meta-analysis. ${ }^{12}$ Of note, Bennett et al also analyzed 22 studies that included women who were overweight and obese, and across these studies, no intervention category significantly reduced the risk of developing GDM. ${ }^{10}$

To understand the mechanisms of action for exercise leading to GDM prevention, Makaruk and colleagues systematically reviewed 10 randomized controlled trials from 5 countries to discuss the effectiveness of exercise during pregnancy by comparing factors that most significantly contributed to GDM risk reduction. ${ }^{13}$ Factors reviewed included frequency, intensity, duration, and type of exercise, as well as adherence and supervision. The initiation of exercise ranged from 6 to 22 weeks gestation, with the majority starting between 9 and 15 weeks gestation and all lasting to the end of pregnancy. Only 2 studies found exercise to be effective in preventing GDM. Both of these had earlier exercise initiation in pregnancy (10-14 weeks gestation), thus allowing for longer total duration of protocol and more sessions completed (72-90), and also had high adherence from participants who performed the exercise regimens 3 times per week for 40-60 minutes. The other 8 studies had lower adherence, shorter duration, and later start dates, all possible contributors to their ineffectiveness in preventing GDM. ${ }^{13}$ The findings from this review echo the conclusions from the previous studies, which all emphasize the importance of early and consistent exercise intervention for successful GDM prevention.

\section{Conclusions}

Synthesis of current literature for this Clin-IQ question shows that exercise during pregnancy is associated with a reduction in risk for gestational diabetes mellitus. These findings affirm the current guidelines from professional groups that recommend exercise during pregnancy to prevent GDM - the American College of Obstetricians and Gynecologists, for example, calls for 30-60 minutes of moderately intense exercise 3-4 times per week in a program that the participant enjoys and therefore is more likely to adhere over the course of the pregnancy. The actual type of exercise can include strength training, moderate aerobic activities, or a combination of both, as current evidence does not specify superiority of one exercise modality over others in preventing GDM. One aspect of the research that was consistent across studies is that starting an exercise program earlier in pregnancy, even prior to pregnancy, decreases the risk of GDM. Consistent exercise should be discussed by clinicians at well-woman exams, prepregnancy counseling visits, and in the first trimester of pregnancy to optimize risk reduction of GDM. 


\section{Patient-Friendly Recap}

- For this Clin-IQ, the authors reviewed recently published studies of the effects of exercise during pregnancy on the development of gestational diabetes.

-While clinically managed exercise alone did not always prove successful, one consistent finding across the literature was that starting an exercise program earlier in (or prior to) pregnancy was noticeably more effective in preventing gestational diabetes.

- Clinicians should discuss exercise during prepregnancy counseling or the first trimester to optimize its benefits.

\section{Acknowledgments}

The authors wish to acknowledge the contribution of Elizabeth Langthorn, MD, MPH, to the initial literature review and preparation of the draft manuscript.

\section{Author Contributions}

Study design: Lust, Chongsuwat, Chou, Wickersham. Data acquisition or analysis: all authors. Manuscript drafting: all authors. Critical revision: all authors.

\section{Conflicts of Interest}

None.

\section{References}

1. Mijatovic-Vukas J, Capling L, Cheng S, et al. Associations of diet and physical activity with risk for gestational diabetes mellitus: a systematic review and meta-analysis. Nutrients. 2018;10(6):698. CrossRef

2. Wilmot EG, Mansell P. Diabetes and pregnancy. Clin Med (Lond). 2014;14:677-80. CrossRef

3. Chatzakis C, Goulis DG, Mareti E, et al. Prevention of gestational diabetes mellitus in overweight or obese pregnant women: a network meta-analysis. Diabetes Res Clin Pract. 2019;158:107924. CrossRef

4. Cremona A, O'Gorman C, Cotter A, Saunders J, Donnelly A. Effect of exercise modality on markers of insulin sensitivity and blood glucose control in pregnancies complicated with gestational diabetes mellitus: a systematic review. Obes Sci Pract. 2018;4:455-67. CrossRef
5. ACOG Committee Opinion, Number 804. Physical activity and exercise during pregnancy and the postpartum period. Obstet Gynecol. 2020;135:e178-88. CrossRef

6. Dipietro L, Evenson KR, Bloodgood B, et al. Benefits of physical activity during pregnancy and postpartum: an umbrella review. Med Sci Sports Exerc. 2019;51:1292-302. CrossRef

7. Griffith RJ, Alsweiler J, Moore AE, et al. Interventions to prevent women from developing gestational diabetes mellitus: an overview of Cochrane Reviews. Cochrane Database Syst Rev. 2020;6(6):CD012394.

8. Guo XY, Shu J, Fu XH, et al. Improving the effectiveness of lifestyle interventions for gestational diabetes prevention: a meta-analysis and meta-regression. BJOG. 2019;126:311-20. CrossRef

9. Ming WK, Ding W, Zhang CJP, et al. The effect of exercise during pregnancy on gestational diabetes mellitus in normalweight women: a systematic review and meta-analysis. $B M C$ Pregnancy Childbirth. 2018;18(1):440. CrossRef

10. Bennett CJ, Walker RE, Blumfield ML, et al. Interventions designed to reduce excessive gestational weight gain can reduce the incidence of gestational diabetes mellitus: a systematic review and meta-analysis of randomised controlled trials. Diabetes Res Clin Pract. 2018;141:69-79. CrossRef

11. Davenport MH, Ruchat SM, Poitras VJ, et al. Prenatal exercise for the prevention of gestational diabetes mellitus and hypertensive disorders of pregnancy: a systematic review and meta-analysis. Br J Sports Med. 2018;52:1367-75. CrossRef

12. Nasiri-Amiri F, Sepidarkish M, Shirvani MA, Habibipour P, Tabari NSM. The effect of exercise on the prevention of gestational diabetes in obese and overweight pregnant women: a systematic review and meta-analysis. Diabetol Metab Syndr. 2019;11:72. CrossRef

13. Makaruk B, Galczak-Kondraciuk A, Forczek W, Grantham W, Charmas M. The effectiveness of regular exercise programs in the prevention of gestational diabetes mellitus - a systematic review. Obstet Gynecol Surv. 2019;74:303-12. CrossRef

14. Schummers L, Hutcheon JA, Bodnar LM, Lieberman E, Himes KP. Risk of adverse pregnancy outcomes by prepregnancy body mass index: a population-based study to inform prepregnancy weight loss counseling. Obstet Gynecol. 2015;125:133-43. CrossRef

(C) 2021 Advocate Aurora Health, Inc. 